Rodríguez-Ruiz, B., Martínez-González, R.A. \& Ceballos-Vacas, E.M. (2019). Las tutorías con las familias en Educación Secundaria Obligatoria: Percepción de padres, madres y profesorado tutor. Revista Electrónica Interuniversitaria de Formación del Profesorado, 22(3), 31-43.

DOI: http://dx.doi.org/10.6018/reifop.22.3.389351

\title{
Las tutorías con las familias en Educación Secundaria Obligatoria: Percepción de padres, madres y profesorado tutor
}

Beatriz Rodríguez-Ruiz', Raquel-Amaya Martínez-González y Esperanza-María CeballosVacas $^{2}$

(1) Universidad de Oviedo, ${ }^{(2)}$ Universidad de La Laguna

\section{Resumen}

En este estudio se analiza la percepción de los padres, las madres y el profesorado tutor sobre quién toma la iniciativa de sus encuentros y los motivos para ello. Han participado 440 padres y 440 madres de las mismas unidades familiares con hijos e hijas en Educación Secundaria Obligatoria (ESO), y 35 tutores y tutoras de este alumnado. La recogida de información se realizó en las comunidades autónomas de Asturias y Canarias con la Escala de Relaciones entre la Familia y el Centro Escolar (Martínez-González, 1994). Los análisis comparativos realizados a través de la t de Student entre la perspectiva de cada uno de los progenitores/as y los tutores/as indican que todos los colectivos coinciden en señalar que contactan con poca frecuencia, y que cuando lo hacen es para comentar, en general, los progresos académicos de sus hijos e hijas. Los aspectos familiares y personales del alumnado, y la organización de actividades conjuntas no suelen ser motivo de las reuniones tutoriales. De ello se pueden extraer orientaciones y sugerencias de actuación.

Palabras clave

Tutoría con Familias; Educación Secundaria Obligatoria; Iniciativa de Contacto; Motivos de Contacto.

\section{Tutoring with families in Compulsory Secondary Education: Perception of fathers, mothers and tutor teachers}

\section{Abstract}

This study analyzes the perception of fathers, mothers and the tutor teachers about who takes the initiative of their meetings and the reasons for it. 440 fathers and 440 mothers of 
the same family unit with children in Compulsory Secondary Education (ESO), and 35 tutors of these students participated. The information was gathered in the autonomous communities of Asturias and the Canary Islands through the Scale on School-Family Relationship (Martínez-González, 1994). The comparative analyses between the perspective of each of the parents and the tutors were carried out through the Student's t, indicating that all the groups agree that they contact with little frequency, and when they do it is to comment, in general, on the academic progress of the children. Interest on the family and on other personal aspects of the students, and on organizing activities together with the parents are not usually reasons for tutorial meetings. From these results, guidelines and suggestions for action can be drawn.

\section{Key words}

Tutoring with Families; Compulsory Secondary Education; Contact Initiative; Reasons for Contact.

\section{Introducción}

La colaboración entre el centro escolar y la familia constituye para la Organización para la Cooperación y el Desarrollo Económico (OCDE) un indicador de calidad educativa (Galián Nicolás, García Sanz y Belmonte Almagro, 2018; Repáraz-Abaitua y Jiménez-García, 2015) que potencia el éxito escolar del alumnado (Consejo Escolar, 2015; Epstein, 2011; Garreta-Bochaca, 2017). De acuerdo con la LOE (Ley Orgánica 2/2006, de 3 de mayo, de Educación) y la LOMCE (Ley Orgánica 8/2013, de 9 de diciembre, para la Mejora de la Calidad Educativa), la iniciativa de establecer esta colaboración recae en los centros escolares, quienes "promoverán compromisos educativos entre las familias o tutores legales y el propio centro en los que se consignen las actividades que padres, profesores y alumnos se comprometen a desarrollar». Para ello ambos agentes han de concebir el centro como un espacio de encuentro, comunicación y participación (Cano y Casado, 2015; CastroZubizarreta y García-Ruiz, 2013)

No obstante, no siempre resulta fácil establecer esta colaboración, en parte por la escasa tradición de participación de las familias en los centros (Rodríguez-Ruiz, ÁlvarezBlanco, Martínez-González y Epstein, 2019), los obstáculos para conciliar la vida laboral, personal y familiar (Rodríguez-Ruiz, Martínez-González, y Rodrigo-López, 2015), o la actitud poco receptiva hacia las familias en algunos centros (Consejo Escolar del Estado, 2015; Usategui-Basozabal y del Valle-Loroño, 2009). De esta forma, la participación de las familias se encuentra supeditada a factores asociados a la cultura organizativa de los centros (clima de confianza general, talante del equipo directivo de apertura a la comunidad...) y al voluntarismo de algunas familias muy motivadas (Martín-Bris y Gairín-Sallán, 2007).

En este complejo reto, el profesorado tutor adquiere una especial relevancia por ser el encargado de iniciar y mantener esta relación y cooperación con las familias, orientarlas, y facilitar el intercambio de información (Torrego-Seijo, 2014), propiciando, según Gil-Villa (1994), más del 50\% de las visitas de las familias a los centros. En esta misma línea, GiróMiranda, Mata-Romeu, Vallespir-Soler, y Vigo-Arrazola (2014) afirman que la forma de participación familiar más exitosa es la tutoría individual, especialmente en secundaria (Andrés-Cabello y Giró-Miranda, 2016), donde la participación de las familias es más reducida (Rodríguez-Ruiz et al., 2015).De hecho, Alguacil-de Nicolás y Pañellas-Valls (2009) encuentran que las familias participan más cuando encuentran en el centro alguien con quien sientan proximidad, como el profesorado tutor. 


\section{Acción tutorial con las familias}

La acción tutorial constituye un elemento central de la función educativa que, desde la LOGSE (Ley Orgánica General del Sistema Educativo, de 3 de octubre de 1990), es asumida por todo el profesorado, en especial, por aquel designado tutor o tutora de un grupo. La tutoría se concibe como una actividad propia de la función docente, y debe desarrollarse de manera individual y colectiva con el alumnado para promover su desarrollo integral; potenciando además las relaciones grupo-clase, grupo-profesorado, profesoradoprofesorado y profesorado-familias (González-Benito, Vélaz de Medrano y López-Martín, 2018). Por tanto, sus responsabilidades incluyen mantener con las familias «una relación fluida, procurando que éstas se impliquen en el proceso de formación del alumno» (SanzOro, 2010, p. 355).La LOE y la LOMCE también resaltan esta colaboración estrecha con las familias, estableciendo la obligación de proporcionarles información periódica sobre el proceso de aprendizaje de sus hijas e hijos, así como orientación para cooperar en el trabajo cotidiano del alumnado y en la vida de los centros escolares. Las normativas autonómicas de las comunidades asturiana y canaria en las que se ubica este estudio regulan más específicamente la acción tutorial con las familias. El Decreto 147/2014, de 23 de diciembre, por el que se regula la Orientación Educativa y Profesional en el Principado de Asturias, refuerza el papel de las familias, promoviendo que el profesorado tutor favorezca la integración, el seguimiento y el acompañamiento del alumnado y sus familias en los centros educativos. Del mismo modo, en Canarias, la Ley 6/2014, de 25 de julio, de Educación no Universitaria, enfatiza la participación de las familias en el proceso educativo de sus hijos e hijas, preocupándose porque la administración, los centros y los servicios educativos faciliten a las familias de forma periódica y adecuada la información y orientación necesaria para estimular su participación. Por su parte, el Decreto 63/2001, de 5 de julio, por el que se aprueba el Reglamento Orgánico de los Centros de Educación Básica del Principado de Asturias señala que el profesorado tutor debe llevar a cabo el plan de acción tutorial con las familias informándoles de las actividades docentes y complementarias y del rendimiento académico, así como facilitando la cooperación educativa. De modo muy similar se expresa el Decreto 81/2010, de 8 de julio, por el que se aprueba el Reglamento Orgánico de los centros docentes públicos no universitarios en Canarias, estableciendo, además, la obligación de realizar con las familias entrevistas periódicas al menos una vez al trimestre.

Por su parte, las familias también han de asumir su responsabilidad de acudir a estas reuniones en el centro; de acuerdo con el profesorado, su asistencia a las tutorías programadas es del $89 \%$ en educación primaria y, aunque disminuye en la ESO, alcanza un $82,4 \%$ en las tutorías individuales en los centros públicos, y un $97,9 \%$ en los privados (ComasSàbat, Escapa-Solanas y Abellán-Cano, 2014). Entre las diversas acciones y temáticas que se tratan en las tutorías, el profesorado admite que la orientación familiar y la personal al alumnado es la que menos realizan (González-Benito et al., 2018); quizás, porque el profesorado, en general, carece de formación para trabajar con las familias (Giró-Miranda y Andrés-Cabello, 2016; Epstein, 2011). Esta situación puede condicionar su disposición a contactar con ellas y los motivos para hacerlo; así como el que las familias inicien estos encuentros. De cualquier forma, las tutorías son el principal medio de comunicación de las familias con el profesorado tutor (Giró-Miranda et al., 2014; Garreta-Bochaca, 2014), si bien es este último quien suele tomar la iniciativa de contactar, por encima de la frecuencia con la que la hacen las familias (Repáraz-Abitua y Jiménez-García, 2015).

En cuanto a los motivos de las tutorías, los más frecuentes son problemas de rendimiento o de comportamiento de las hijas y los hijos (Garreta-Bochaca, 2015; MartínezGonzález et al, 2002), especialmente en educación secundaria (Giró-Miranda et al., 2014). No obstante, García-Sanz, Gomariz-Vicente, Parra-Martínez y Hernández-Prados (2010) 
constatan que también se centran en aspectos positivos y personales del proceso educativo (desarrollo personal, salud...); aunque las familias tienden más a juzgar que se presta poca atención a estos aspectos (Repáraz-Abitua y Jiménez-García, 2015). Sea como sea, el seguimiento escolar del alumnado y el apoyo familiar en las tareas escolares parecen ser las temáticas más recurrentes (García-Bacete, 2006).

En el presente estudio se pretende profundizar en estos aspectos, contrastando las opiniones que tienen, tanto el profesorado tutor como las madres y los padres, sobre la frecuencia de quién promueve las tutorías durante la ESO y de sus motivos para estos contactos.

\section{Metodología}

El objetivo principal de este estudio es analizar la percepción de los padres, las madres y el profesorado tutor sobre quién toma la iniciativa para promover las tutorías individuales. Se pretende, así mismo, identificar los principales motivos por los cuales se producen los contactos entre las familias y el profesorado tutor, en la etapa de la ESO. Se parte de la hipótesis de que los contactos son escasos, centrándose principalmente en aspectos académicos o problemáticos del alumnado.

\section{Participantes}

En el estudio participan 880 personas de la misma unidad familiar, 440 padres y 440 madres con adolescentes escolarizados en $2^{\circ}$ y $4^{\circ}$ de ESO, y 35 tutoras y tutores de sus hijos e hijas. Así pues, por cada adolescente se ha recogido información tanto de ambos progenitores como de su tutor o tutora, lo que suele ser poco habitual en los estudios de encuesta. El perfil sociodemográfico de los padres y madres es de nacionalidad española (94,4\%), con una edad media de 45,51 años (DT=,54) para el padre (69,5\% entre 41 y 51 años) y de 43,29 años (DT=,54) para la madre (66,1\% entre 41 y 51 años), casados/as ( $92 \%$ padre), con estudios de Bachillerato/Formación Profesional (38,9\% padres y 40,7\% madres), y con trabajo fuera del hogar familiar ( $92,5 \%$ padres 46 y $67,5 \%$ madres), si bien un $25,9 \%$ de las madres trabajan de manera exclusiva no remunerada en el hogar familiar. Su residencia se distribuye en zonas urbanas $(45,2 \%)$ y rurales $(54,8 \%)$ de las comunidades autónomas de Asturias (varios municipios: Oviedo, Mieres, Noreña, etc., 57,5\%) y Canarias (varios municipios de Tenerife $45,2 \%)$.

El profesorado ( $54,5 \%$ tutoras y $45,5 \%$ tutores) tiene una edad media de 42,09 años (53,4\% entre 40-48; DT = ,65), y una experiencia media como tutoras y tutores de 14,95 años (DT= ,86). El 55\% imparte asignaturas del ámbito socio-lingüístico y el $45 \%$ del científicotecnológico.

\section{Instrumento y procedimiento}

La recogida de información se llevó a cabo con la Escala de Relaciones entre el Centro Escolar y la Familia, adaptada por Martínez-González (1994) tanto en la versión para los padres y las madres como para el profesorado, del original Family-School Relationship Trust Scale, del Partneship for School Success Program (Sinclair, Lam, Christenson y Evelo, 1993). Todas las versiones constan de 64 ítems correspondientes a cinco dimensiones: Percepción de las familias sobre la colaboración del profesorado; Contactos entre el profesorado tutor y las familias; Estudios del hijo/a e implicación de la familia; Relación con el centro escolar; y Participación familiar en el centro escolar.

En este estudio se analizan los cinco indicadores que configuran la dimensión Contactos entre el profesorado tutor y las familias, con redacción adaptada en los ítems de 
cada versión, y respuestas de escala Likert con cinco puntos de frecuencia (1-Nunca; 2-Pocas veces; 3-Algunas veces; 4-A menudo; 5-Siempre). En estos ítems se analizan los motivos de los contactos: 1) informar sobre los progresos escolares del hijo/a; 2) comunicar algún aspecto positivo del hijo/a; 3) comentar problemas; 4) interés por cuestiones personales del hijo/a o de la familia (salud, ausencias de clase, etc.); y 5) organizar actividades en colaboración con la familia. Un ejemplo de ítem de la versión para los padres o las madres es: Durante el pasado curso, o en este, el tutor/a contactó conmigo para comunicarme algún aspecto positivo del comportamiento de mi hijo/a. En los tres primeros indicadores se analiza la percepción del padre, la madre y el tutor o la tutora sobre quién tomó la iniciativa para el contacto (docente o el padre o la madre) contando, por tanto, con seis medidas por indicador (ver Tabla 1). En los dos últimos se cuenta únicamente con tres medidas, ya que sólo se analiza la percepción de cada colectivo sobre si el tutor o la tutora emprendió la iniciativa para el encuentro (ver Tabla 2).

La estructura factorial de la versión de la escala para padres obtuvo una medida Kayser-Meyer-Olsen=,875 y un valor significativo en la prueba de esfericidad de Bartlett $\chi(1596)=12405,766 ; p=, 000$, con una varianza explicada del 57,40\%. En la versión para las madres se obtuvo una medida Kayser-Meyer-Olsen=,848 y un valor significativo en la prueba de esfericidad de Bartlett $\chi(1596)=10970,487 ; p=, 000$, con una varianza explicada del $49,72 \%$. Por su parte, la versión para el profesorado tutor obtuvo una medida KayserMeyer-Olsen=,875 y un valor significativo en la prueba de esfericidad de Bartlett $\chi(1345)=1459,598 ; p=, 000$, con una varianza explicada del 73,03\%. El índice de fiabilidad de la dimensión analizada es de $\alpha=, 86$ (padres), $\alpha=, 83$ (madres), y $\alpha=, 88$ (profesorado tutor); todos ellos valores adecuados en ciencias sociales (Elosua Oliden y Zumbo, 2008).

Se contactó por carta con 6 centros escolares de diversos municipios de Asturias y 4 centros escolares de Tenerife, solicitando su colaboración. Tras su aceptación se envió a las familias y al profesorado tutor del alumnado de $2^{\circ}$ y $4^{\circ}$ de ESO otra carta en la que se les pedía que cumplimentaran el instrumento (con instrucciones muy detalladas sobre cómo responder) y firmaran un protocolo de consentimiento, garantizando su anonimato. El procedimiento fue aprobado por el Comité de Ética de la Investigación y de Bienestar Animal (CEIBA) de la Universidad de La Laguna. Se obtuvieron autorizaciones de las familias en un $95 \%$ de los casos, si bien no todas ellas cubrieron finalmente la escala a aplicar. El porcentaje final recogido resultó del $85 \%$.

\section{Análisis de datos}

Se calcularon con el programa SPSS.22 estadísticos descriptivos de frecuencias, porcentajes y medidas de tendencia central y variabilidad para cada ítem, así como su asimetría y curtosis, considerando valores [-2; +2], respectivamente, para comprobar si su distribución se ajustaba a la curva normal (George y Mallery, 2010). Posteriormente, se realizaron análisis comparativos de dos grupos independientes con la $t$ de Student para identificar diferencias significativas al comparar las perspectivas del profesorado tutor y de los padres y las madres en sus apreciaciones sobre quién inicia los contactos para las tutorías y los motivos para hacerlo. En cada análisis se tuvo en cuenta la condición de homogeneidad de las varianzas (prueba de Levene). Cuando las diferencias entre grupos resultaron significativas se calculó el tamaño del efecto con la d de Cohen, interpretando su magnitud de acuerdo con los siguientes criterios (Cohen, 1988): tamaño bajo $0<d<, 20$; mediomoderado, $20<d \leq, 50$; y alto $d>, 50$, si bien se tuvo en cuenta que incluso un tamaño del efecto pequeño puede tener una significación práctica (Kirk, 1996). 


\section{Resultados}

\section{Percepción de los padres y las madres sobre los contactos con el profesorado tutor}

Los análisis descriptivos de la percepción de los padres y las madres acerca de su relación con el profesorado tutor (tabla 1) indican que las familias estiman que contactan poco, independientemente de que consideren que las tutorías han sido promovidas por el profesorado o por los propios padres y madres. En concreto, el valor medio de frecuencia se halla, en general, entre pocas y algunas veces, lo que da cuenta de las escasas ocasiones en las que se encuentran las familias y el profesorado tutor.

Tabla 1.

Descriptivos y contrastes en cada colectivo sobre la percepción de iniciativa para los contactos y su frecuencia

\begin{tabular}{|c|c|c|c|c|c|c|}
\hline Indicadores & Colectivo & $\begin{array}{l}\text { Percepción sobre } \\
\text { quién inicia } \\
\text { contacto }\end{array}$ & $\begin{array}{c}\text { M (DT) percepción } \\
\text { de frecuencia de } \\
\text { contacto }\end{array}$ & $t$ & $p$ & $d$ \\
\hline \multirow{6}{*}{$\begin{array}{l}\text { Informar sobre los } \\
\text { progresos escolares del } \\
\text { hijo/a }\end{array}$} & \multirow{2}{*}{ Padres } & Profesorado tutor & $2,65(1,45)$ & \multirow{2}{*}{$-5,805$} & \multirow{2}{*}{,000 } & \multirow{2}{*}{$-0,297$} \\
\hline & & Padre & $3,07(1,37)$ & & & \\
\hline & \multirow{2}{*}{ Madres } & Profesorado tutor & $2,74(1,41)$ & \multirow{2}{*}{$-1,455$} & \multirow{2}{*}{,146 } & \multirow{2}{*}{-} \\
\hline & & Madre & $2,85(1,37)$ & & & \\
\hline & \multirow{2}{*}{ Profesorado tutor } & Profesorado tutor & $2,81(1,81)$ & \multirow{2}{*}{2,926} & \multirow{2}{*}{,004 } & \multirow{2}{*}{0,105} \\
\hline & & Padre/madre & $2,65(1,14)$ & & & \\
\hline \multirow{6}{*}{$\begin{array}{l}\text { Comunicar algún } \\
\text { aspecto positivo del } \\
\text { hijo/a }\end{array}$} & \multirow{2}{*}{ Padres } & Profesorado tutor & $2,42(1,42)$ & \multirow{2}{*}{$-2,414$} & \multirow{2}{*}{,016 } & \multirow{2}{*}{$-0,114$} \\
\hline & & Padre & $2,58(1,38)$ & & & \\
\hline & \multirow{2}{*}{ Madres } & Profesorado tutor & $2,48(1,40)$ & \multirow{2}{*}{$-3,448$} & \multirow{2}{*}{,001 } & \multirow{2}{*}{$-0,161$} \\
\hline & & Madre & $2,70(1,32)$ & & & \\
\hline & \multirow{2}{*}{ Profesorado tutor } & Profesorado tutor & $2,54(1,15)$ & \multirow{2}{*}{, 460} & \multirow{2}{*}{,646 } & \multirow{2}{*}{-} \\
\hline & & Padre/madre & $2,51(1,13)$ & & & \\
\hline \multirow{6}{*}{$\begin{array}{c}\text { Comentar problemas } \\
\text { del hijo/a }\end{array}$} & \multirow{2}{*}{ Padres } & Profesorado tutor & $2,28(1,33)$ & \multirow{2}{*}{$-5,002$} & \multirow{2}{*}{, 000} & \multirow{2}{*}{$-0,231$} \\
\hline & & Padre & $2,59(1,35)$ & & & \\
\hline & \multirow{2}{*}{ Madres } & Profesorado tutor & $2,40(1,35)$ & \multirow{2}{*}{$-1,956$} & & \\
\hline & & Madre & $2,53(1,29)$ & & ,051 & - \\
\hline & Dreforeno do & Profesorado tutor & $2,20(1,10)$ & & & 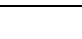 \\
\hline & Protesorado tutor & Padre/madre & $2,25(1,08)$ &,- 982 & ,326 & - \\
\hline Interesarse por & Padres & Profesorado tutor & $2,28(1,38)$ & - & - & - \\
\hline cuestiones personales & Madres & Profesorado tutor & $2,15(1,28)$ & - & - & - \\
\hline $\begin{array}{c}\text { del alumno/a o de la } \\
\text { familia }\end{array}$ & Profesorado tutor & Profesorado tutor & $2,19(1,09)$ & - & - & - \\
\hline Organizar actividades & Padres & Profesorado tutor & $1,79(1,05)$ & - & - & - \\
\hline en colaboración con la & Madres & Profesorado tutor & $1,90(1,14)$ & - & - & - \\
\hline familia & Profesorado tutor & Profesorado tutor & $2,00(1,09)$ & - & - & - \\
\hline
\end{tabular}

Los motivos que los padres ven como más habituales en el profesorado para establecer contacto con las familias son informar sobre los progresos escolares de sus hijos/as $(M=2,65 ; D T=1,45)$ y comunicarles algún aspecto positivo $(M=2,42 ; D T=1,42)$, ambos ubicados entre las frecuencias pocas veces y algunas veces. Otros motivos como comentar problemas de los hijos/as $(M=2,28 ; D T=1,33)$ o interesarse por cuestiones personales o familiares $(M=2,28$; $D T=1,38)$ cuentan con una frecuencia media menor, alcanzando un valor algo por encima de pocas veces. No obstante, los padres consideran que el motivo por el que el profesorado programa una tutoría con menor frecuencia es la organización de actividades en colaboración con la familia $(M=1,79 ; D T=1,05)$, lo que, según ellos, ocurre entre nunca y pocas veces.

Los padres perciben esta misma tendencia a la baja frecuencia cuando la iniciativa para los encuentros parte de ellos mismos, excepto respecto a informarse sobre los 
progresos escolares de sus hijo/as $(M=3,07 ; D T=1,37)$, cuya media llega a algunas veces, constituyendo la frecuencia más elevada en los tres colectivos. Así mismo, se aprecian diferencias significativas a favor de esta percepción cuando se la compara con la que sostienen acerca de la frecuencia con la que el profesorado tutor emprende un contacto por esta misma causa, con un tamaño del efecto medio $(p=, 000 ; d=-, 297)$. Por su parte, la percepción sobre sus iniciativas para pedir una tutoría para comentar problemas $(M=2,59$; $D T=1,35)$, y comunicar algún aspecto positivo de sus hijos/as $(M=2,58 ; D T=1,38)$ muestra frecuencias similares. En ambos casos se obtienen, además, diferencias significativas al comparar su percepción de la frecuencia sobre quién promueve los contactos (ellos mismos o el profesorado tutor), manifestándose de nuevo una tendencia a su favor respecto a los motivos comunicar aspectos positivos $(p=, 016 ; d=-, 114)$ y comentar problemas de sus hijos/as ( $p=, 000 ; d=-, 231)$, con unos tamaños del efecto bajo y medio, respectivamente.

La perspectiva de las madres es muy parecida a la de los padres, si bien con una tendencia a percibir que las reuniones se producen con una frecuencia relativamente superior en las motivaciones estudiadas. Cuando las madres estiman que las tutorías son promovidas por el profesorado creen que los motivos más usuales (entre pocas veces y algunas veces) para que las convoquen son: informar sobre los progresos escolares de sus hijos/as ( $M=2,74 ; D T=1,41)$, comunicarles aspectos positivos de sus hijos/as ( $M=2,48 ; D T=1,40)$, y comentar problemas que afectan a sus hijos/as $(M=2,40 ; D T=1,35)$. Sin embargo, el interés por los aspectos personales de los hijos/as o de la familia $(M=2,15 ; D T=1,28)$ apenas supera la frecuencia pocas veces. Por fin, la organización de actividades en colaboración con la familia $(M=1,90 ; D T=1,14)$ es considerada una motivación atípica en el profesorado tutor, ya que su media ni siquiera llega a la frecuencia pocas veces.

En cuanto a la percepción de los motivos esgrimidos para propiciar un contacto por parte de las propias madres, en primer lugar, aparece de nuevo informarse sobre el progreso escolar de sus hijos/as ( $M=2,85 ; D T=1,37)$, seguido por comunicar aspectos positivos de sus hijos/as $(M=2,70 ; D T=1,32)$, y comentar problemas de sus hijos/as $(M=2,53 ; D T=1,29)$.Todas estas motivaciones se aproximan a la frecuencia algunas veces. Así mismo, se obtuvieron diferencias significativas al comparar su percepción sobre quién inicia los contactos (ellas mismas o el profesorado tutor) respecto al motivo comunicar aspectos positivos de sus hijos/as, revelándose una tendencia a su favor, con un tamaño del efecto bajo ( $p=, 001 ; d=-$ ,161).

\section{Percepción del profesorado tutor sobre los contactos con las familias}

Los análisis descriptivos (ver Tabla 1) indican que, en términos generales, los tutores y las tutoras también perciben una baja frecuencia de contacto con las familias. De esta manera informan de que cuando emprenden la iniciativa de una tutoría, se centran, en primer lugar, en informar a las familias sobre los progresos escolares de sus hijos/as $(M=2,81 ; D T=1,81$,$) y en$ comunicarles aspectos positivos $(M=2,54 ; D T=1,15)$, con una frecuencia que se acerca a algunas veces. A continuación, consideran que otros motivos usuales son comentarles problemas de sus hijos/as $(M=2,20 ; D T=1,10)$ e interesarse por cuestiones personales del alumnado $y / 0$ de sus familias $(M=2,19 ; D T=1,09)$, que se sitúan algo por encima de pocas veces. El motivo con menor frecuencia percibida es, nuevamente, la organización de actividades en colaboración con las familias $(M=2,00 ; D T=1,09)$ cuya media corresponde a pocas veces.

Respecto a las ocasiones en las que el profesorado tutor entiende que son las familias quienes promueven las tutorías, piensan que las causas más habituales (que se dan entre pocas veces y algunas veces) son, en este orden, informarse sobre los progresos escolares de sus hijos/as $(M=2,65 ; D T=1,14)$, comunicar algún aspecto positivo de su 
comportamiento $(M=2,51 ; D T=1,13)$, y comunicar algún problema de sus hijos/as $(M=2,25$; $D T=1,08)$.

Al comparar la percepción de los tutores y las tutoras sobre quién favorece los contactos (el profesorado o los padres/madres), también se encontraron diferencias significativas a su favor respecto al motivo informar sobre los progresos escolares de los hijos/as, con un tamaño del efecto bajo $(p=, 004 ; d=-, 105)$.

Contrastes entre las perspectivas de los padres, las madres y el profesorado tutor sobre quién inicia los contactos y sus motivos

Los análisis comparativos entre las perspectivas del profesorado tutor y de los padres (ver Tabla 2), respecto a los contactos promovidos por los tutores y las tutoras, establecen diferencias significativas en cuanto al motivo organizar actividades en colaboración con las familias, señalando que el profesorado tutor percibe que toma esta iniciativa con mayor frecuencia de la que los padres les atribuyen, con un tamaño del efecto bajo ( $t(439)=-3,038$; $p=, 003 ; d=, 196)$. Por su parte, la comparación entre las percepciones del profesorado tutor y las madres encuentra estas diferencias en el indicador comentar problemas del hijo/a, sugiriendo que las madres valoran que el profesorado solicita encuentros por este motivo con más frecuencia de la que el propio profesorado reconoce, con un tamaño del efecto bajo $(t(439)=-2,538 ; p=, 011 ; d=-, 162)$.

Tabla 2.

Contrastes entre las percepciones del profesorado tutor y los padres y las madres sobre las frecuencias de contactos y sus motivos

\begin{tabular}{|c|c|c|c|c|c|}
\hline Ítems & Colectivos & $M(D T)$ & $t$ & $p$ & $d$ \\
\hline \multirow{2}{*}{$\begin{array}{l}\text { El Tutor/a contactó con la familia para } \\
\text { organizar actividades en colaboración }\end{array}$} & Profesorado tutor & $2,00(1,09)$ & \multirow[b]{2}{*}{3,038} & \multirow[b]{2}{*}{, 003} & \multirow[b]{2}{*}{ 196 } \\
\hline & Padres & $1,79(1,05)$ & & & \\
\hline \multirow{2}{*}{$\begin{array}{l}\text { El Tutor/a contactó con la familia para } \\
\text { comentar algún problema referente al hijo/a }\end{array}$} & Profesorado tutor & $2,20(1,10)$ & \multirow[b]{2}{*}{$-2,538$} & \multirow[b]{2}{*}{,011 } & \multirow[b]{2}{*}{,- 162} \\
\hline & Madres & $2,40(1,35)$ & & & \\
\hline \multirow{4}{*}{$\begin{array}{l}\text { La familia contactó con el tutor/a para } \\
\text { informarse sobre los progresos del hijo/a }\end{array}$} & Profesorado tutor & $2,65(1,13)$ & \multirow{2}{*}{$-2,570$} & \multirow{2}{*}{,011 } & \multirow{2}{*}{,- 158} \\
\hline & Madres & $2,85(1,37)$ & & & \\
\hline & Profesorado tutor & $2,65(1,13)$ & \multirow{2}{*}{$-5,199$} & \multirow{2}{*}{,000 } & \multirow{2}{*}{,- 333} \\
\hline & Padres & $3,07(1,37)$ & & & \\
\hline \multirow{2}{*}{$\begin{array}{c}\text { La familia contactó con el tutor/a para } \\
\text { comentar algún aspecto positivo del } \\
\text { comportamiento del hijo/a }\end{array}$} & Profesorado tutor & $2,51(1,13)$ & \multirow[b]{2}{*}{$-2,414$} & \multirow[b]{2}{*}{,016 } & \multirow[b]{2}{*}{,- 154} \\
\hline & Madres & $2,70(1,32)$ & & & \\
\hline \multirow{4}{*}{$\begin{array}{l}\text { La familia contactó con el tutor/a para } \\
\text { comentar algún problema referente al hijo/a }\end{array}$} & Profesorado tutor & $2,25(1,08)$ & \multirow{2}{*}{$-3,722$} & \multirow{2}{*}{,000 } & \multirow{2}{*}{,- 235} \\
\hline & Madres & $2,53(1,29)$ & & & \\
\hline & Profesorado tutor & $2,25(1,08)$ & \multirow{2}{*}{$-4,274$} & \multirow{2}{*}{,000 } & \multirow{2}{*}{,- 278} \\
\hline & Padres & $2,59(1,35)$ & & & \\
\hline
\end{tabular}

Respecto a las temáticas o motivos de las reuniones fomentadas por las familias también se encuentran diferencias significativas entre los tres colectivos: tanto los padres $(t(439)=-5,199 ; p=, 000 ; d=-, 333)$ como las madres $(t(439)=-2,570 ; p=, 011 ; d=-, 158)$ consideran que piden tutorías con mayor frecuencia de la que estima el profesorado para informarse sobre los progresos académicos de sus hijos/as, con un tamaño del efecto medio y bajo, respectivamente. Además, las madres señalan que se comunican para hablar de aspectos positivos de sus hijos/as con mayor frecuencia de la indicada por el profesorado tutor, con un tamaño del efecto bajo $(t(439)=-2,414 ; p=, 016 ; d=-, 154)$. Por último, nuevamente, tanto los padres $(t(439)=-4,274 ; p=, 000 ; d=-, 278)$ como las madres $(t(439)=-3,722 ; p=, 000 ; d=-, 235)$ 
perciben que emprenden más iniciativas de tutorías para comentar problemas de sus hijos/as de las que admite el profesorado tutor, con un tamaño del efecto medio en ambos casos.

\section{Discusión y Conclusiones}

La promoción de relaciones entre el profesorado tutor y las familias se dispone en la normativa educativa como un elemento necesario para facilitar el ajuste académico y personal del alumnado, así como para favorecer la participación general de las familias. Diversos autores han constatado que si bien en la educación infantil y primaria estos contactos se realizan con cierta frecuencia, en la educación secundaria tienden a ser escasos (Andrés-Cabello y Giró-Miranda, 2016; Deslandes y Bertrand, 2005) y, cuando se producen, suelen obedecer a problemas académicos o de comportamiento del alumnado (Repáraz-Abitua y Jiménez-García, 2015). Esta concentración en aspectos negativos puede condicionar las iniciativas de familias y docentes, así como sus actitudes en estas reuniones. Por ello, el objetivo de este estudio ha sido contrastar la visión que tienen los padres, las madres y el profesorado tutor de un mismo alumno o alumna sobre quién toma la iniciativa de contactar en función de los motivos que suscitan dicha decisión, y la medida en la que lo hacen.

Los resultados confirman esta tendencia a la baja frecuencia de contacto, ya que tanto el profesorado tutor como los padres y las madres, señalan que mantienen tutorías pocas o algunas veces, ya sean fomentadas por el profesorado o por las familias. Estos resultados han sido también resaltados por Comas-Sàbat et al. (2014), subrayando la reducción de reuniones individuales entre el profesorado tutor y las familias en la ESO (Rodríguez-Ruiz et al., 2015), con independencia de quién tome la iniciativa para entablar estos encuentros. Esta situación resulta preocupante en una etapa educativa en la que el alumnado precisa de un apoyo decidido y conjunto del profesorado y su familia para orientarle, tanto respecto a aspectos académicos, como personales y vocacionales (SanzOro, 2010).

El análisis de las perspectivas de los padres y de las madres sobre quiénes impulsan más los contactos (si las familias o el profesorado tutor), indica que los padres sostienen que ellos son quienes toman la iniciativa significativamente más (en comparación con el profesorado tutor) tanto para tratar aspectos positivos como problemas de sus hijos e hijas. Las madres también estiman que solicitan significativamente más reuniones con el profesorado para comunicar aspectos positivos de sus hijos/as. Por su parte, el profesorado tutor percibe que inicia los contactos significativamente más que los padres o las madres para informar sobre los progresos de los hijos e hijas, lo que es coherente con su función docente.

Al contrastar las opiniones entre los tres colectivos, en cuanto a los motivos que suscitan las tutorías, se observa que todos parecen estar de acuerdo en que el primordial es comentar los progresos académicos del hijo o hija, en sintonía con lo ya referido en otros estudios (García-Bacete, 2006). Este dato también encaja con la individualización característica de las relaciones entre los centros escolares y las familias en secundaria, ya que las madres y los padres están más concentrados en el seguimiento de las calificaciones de sus hijas e hijos por la importancia en su futura trayectoria académica (Giró-Miranda y Andrés-Cabello, 2016). Por otro lado, a pesar de que en secundaria se incida en las causas relacionadas con problemas de los hijos e hijas (Garreta-Bochaca, 2015; Giró-Miranda et al., 2014), en este estudio todos los colectivos han tendido a destacar, como segundo motivo de contacto, hablar sobre aspectos positivos; este halagüeño resultado también ha sido subrayado por García-Sanz et al. (2010). En cambio, los contenidos menos aludidos han sido los aspectos personales o familiares del alumnado, de acuerdo con lo apuntado por 
Repáraz-Abitua y Jiménez-García (2015), así como la organización conjunta de actividades. Todo ello pondría de manifiesto que las tutorías no se suelen aprovechar para estimular la participación general de las familias en el centro.

En cuanto a quién promueve más las tutorías, el análisis de discrepancias entre colectivos sugiere que las familias se perciben con mayor grado de iniciativa del que le atribuye el profesorado. En concreto, los padres y las madres estiman significativamente mayor la frecuencia con la que se responsabilizan de fomentar reuniones para informarse sobre los progresos académicos y comentar tanto aspectos positivos como problemas de sus hijos e hijas respecto a la que le arroga el profesorado tutor. Este resultado podría sugerir que el interés de las familias por los aspectos escolares de sus hijos e hijas es mayor de lo que tiende a opinar el profesorado (Usategui-Basozabal y del Valle-Loroño, 2009). Por su parte, el profesorado tutor se percibe a sí mismo como promotor principal de estos contactos, tal como indican también Repáraz-Abitua y Jiménez-García (2015), lo que es coherente con su función de convocar periódicamente a las familias. De cualquier forma, y de acuerdo con Bolívar-Botía (2006), estas invitaciones a las familias por parte del profesorado constituyen un factor motivador relevante, que puede contribuir a facilitar su participación.

Atendiendo a las percepciones de las familias sobre los motivos por los que el profesorado les convoca a tutorías, en este estudio se ha encontrado que son las madres quienes más difieren de la perspectiva de las tutoras y los tutores. Así, perciben que son requeridas a tutorías para que les comenten problemas de sus hijos e hijas con mayor frecuencia de la que el profesorado reconoce. Por su parte, los padres discrepan con el profesorado tutor al considerar que estos no invitan tanto a las familias a organizar actividades conjuntas, en comparación con lo declarado por las tutoras y los tutores. De acuerdo con Alguacil-de Nicolás y Pañellas-Valls (2009) y Garreta-Bochaca (2017), esta concentración en los aspectos más espinosos y molestos (suspensos, malas conductas...) en detrimento de los positivos y personales, redunda en que ambos colectivos se vayan situando en una posición de defensa, lo que merma las posibilidades de una comunicación más constructiva y estable (Castro-Zubizarreta, García-Ruiz y Maraver-López, 2018).

Cuando las reuniones son promovidas por parte de las familias, tanto los padres como las madres, consideran que son quienes más abordan el progreso académico, los aspectos positivos y las situaciones problemáticas de sus hijos e hijas en comparación con el profesorado. De cualquier modo, la prevalencia de los contactos para tratar el progreso académico y los aspectos positivos del alumnado resulta un dato para el optimismo respecto a la mejora de las relaciones de las familias con los centros escolares y de su participación. El interés común de las familias y el profesorado tutor por el desarrollo de las y los adolescentes a través de las tutorías, puede contribuir a gestar una alianza provechosa y un clima de confianza favorable. Además, las familias valoran más al profesorado tutor cuando les informa y se implica en el seguimiento de las tareas escolares de sus hijos e hijas (Risko y Walker-Dalhouse, 2009). Y a su vez, el profesorado tutor se siente más competente para contribuir al desarrollo académico y personal del alumnado, cuando cuenta con el apoyo de las familias en su acción tutorial (Jeynes, 2007; Sheldon y Epstein, 2005).

Estos resultados nos animan a seguir defendiendo la necesidad de incentivar los contactos entre las familias y el profesorado en las tutorías. No obstante, será necesario reforzar la preparación del profesorado para cooperar con las familias puesto que, como los y las docentes sostienen, en general, carecen de formación inicial para estimular la participación (Giró-Miranda y Andrés-Cabello, 2016), y para trabajar con las familias (Epstein, 2011). En este sentido, Gomariz-Vicente, Hernández-Prados, García-Sanz y Parra-Martínez, 2017, p. 50) señalan que «los docentes que más formación poseen respecto al trato con las 
familias son los que, significativamente, más facilitan la participación de padres y madres en el centro educativo, tanto en infantil como en primaria y secundaria»; un dato también refrendado por Castro-Morera et al. (2015).

Como posibles líneas de investigación futuras cabe proponer este mismo análisis desde la perspectiva del alumnado de la ESO, ya que su voz puede aportar una visión complementaria a la de la percepción adulta. Así mismo, y con fines preventivos, es interesante ampliar este estudio a la etapa de Educación Primaria, donde los procesos de interacción entre el profesorado tutor y las familias, no están tan influidos por la toma de decisiones del futuro académico, personal y profesional de las niñas y los niños. Por otro lado, sería conveniente disponer de información cualitativa procedente de entrevistas y grupos de discusión para enriquecer y contrastar los datos de este estudio. Por último, sería de interés interpretar estos resultados en función de variables asociadas a los centros escolares, como la titularidad del centro, su cultura escolar acerca de la participación, y su ubicación geográfica en zonas rurales y urbanas.

\section{Referencias}

Alguacil-de Nicolás, Montserrat \& Pañellas-Valls, Mercè (2019). Implicación de las familias en los institutos de enseñanza secundaria. Magis. Revista Internacional de Investigación en Educación, 2(3), 111-127.

Andrés-Cabello, Sergio \& Giró-Miranda, Joaquín (2016). El papel y la representación del profesorado en la participación de las familias en la escuela. Revista Electrónica Interuniversitaria de Formación del Profesorado, 19(1), 61-71.

Bolívar-Botía, Antonio (2006). Familia y escuela: dos mundos llamados a trabajar en común. Revista de educación, 339, 119-146.

Cano, Rufino \& Casado, Mónica (2015). Escuela y familia. Dos pilares fundamentales para unas buenas prácticas de orientación educativa a través de las escuelas de padres. Revista Electrónica Interuniversitaria de Formación del Profesorado, 18 (2), 15 -27.

Castro-Morera, María, Expósito-Casas, Eva, Lizasoain-Hernández, Luis, López-Martín, Esther, \& Navarro-Asencio, Enrique (2015). Acciones y actitudes diferenciales de los tutores y su relación con la participación de las familias. Participación Educativa, 4(7), 29-37.

Castro-Zubizarreta, Ana \& García-Ruiz, María-Rosa (2013). La visión del profesorado de educación infantil y primaria de Cantabria sobre la participación y las relaciones interpersonales entre los miembros de la comunidad escolar. Aula Abierta, 41(1), 7384.

Castro-Zubizarreta, Ana, García-Ruiz, María-Rosa, \& Maraver-López, Pablo (2018). Impacto del practicum en las creencias de los maestros en formación sobre la relación familia-escuela. Revista Brasileira de Educação, 23, 1-19.

Cohen, Jacob (1988). Statistical power analysis for the behavioral science ( $2^{\mathrm{a}}$ ed.). Hillsdale, $\mathrm{NJ}$ : Lawrence Erlbaum Associates.

Comas-Sàbat, Marta, Escapa-Solanas, Sandra \& Abellán-Cano, Carlos (2014). Com participen mares i pares a l'escola? Diversitat familiar i d'implicació en educación. Informes Breus, 48. Bacerlona: Fundació Jaume Bofill.

Consejo Escolar del Estado (2015). La participación de las familias en la educación escolar. Madrid: MECD. 
Deslandes, Rollande \& Bertrand, Richard (2005). Motivation of Parent Involvement in Secondary-level Schooling. The Journal of Educational Research (January-February), 98 (3), 164-175.

Elosua Oliden, Paula \& Zumbo D., Bruno (2008). Coeficientes de fiabilidad para escalas de respuesta categórica ordenada. Psicothema, 20 (4), 896-901.

Epstein, Joyce-L. (2011). School, Family and Community Partnerships. Preparing Educators and Improving Schools. Philadelphia: WESTVIEW Press.

Galián Nicolás, Begoña, García Sanz, Ma-Paz. \& Belmonte Almagro, Mª-Luisa (2018). Evaluación de la participación familiar en el proceso educativo de los discentes desde la percepción del profesorado. Revista Electrónica Interuniversitaria de Formación del Profesorado, 21(3), 45-62.

García-Sanz, Ma-Paz, Gomariz-Vicente, Ma-Ángeles, Hernández-Prados \& $M^{\mathrm{a}}$-Ángeles \& Parra-Martínez, Joaquín (2010). La comunicación entre la familia y el centro educativo, desde la percepción de los padres y madres de los alumnos. Educatio Siglo XXI, 28(1), 157-188.

García-Bacete, Francisco-Juan (2006): Cómo son y cómo podrían ser las relaciones entre escuelas y familias en opinión del profesorado. Cultura y Educación, 18(3-4), 247-265.

Garreta-Bochaca, Jordi (2014). La participación de las familias en la escuela: una cuestión pendiente. Documentación Social. Revista de Estudios Sociales y Sociología Aplicada, 171, 101-124.

Garreta-Bochaca, Jordi (2015). La comunicación familia-escuela en educación infantil y primaria. Revista de la Asociación de Sociología de la Educación, 8(1), 71-85.

Garreta-Bochaca, Jordi (Coord.) (2017). Familias y escuelas. Discursos y prácticas sobre la participación en la escuela. Madrid: Pirámide.

George, Darren \& Mallery, Paul (2010). SPSS for Windows Step by Step: A Simple Guide and Reference, 17.0 update. Boston: Pearson.

Giró-Miranda, Joaquín \& Andrés-Cabello, Sergio (2016). Instalados en la queja: El profesorado ante la participación de las familias en las escuelas. Revista de la Asociación de Sociología de la Educación, 9(3), 334-345.

Giró-Miranda, Joaquín, Mata-Romeu, Anna, Vallespir-Soler, Jordi \& Vigo-Arrazola, Begoña (2014). Familias y escuelas: los diferentes discursos sobre la participación. Ehquidad International Welfare Policies and Social Work Journal, 2, 65-89.

Gomariz-Vicente, $M^{a}$-Ángeles, Hernández-Prados, $M^{a}$-Ángeles, García-Sanz, Ma-Paz \& ParraMartínez, Joaquín (2017). Tejiendo puentes entre la escuela y la familia. El papel del profesorado. Bordón, 69(2), 41-57.

González-Benito, Ana, Vélaz de Medrano-Ureta, Consuelo \& López-Martín, Esther (2018). La tutoría en educación primaria y secundaria en España: una aproximación empírica. REOP, 29(2), 105-127.

Jeynes, William (2007). The relationship between parental involvement and urban secondary school student academic achievement: A meta-analysis. Urban Education, $42,82-110$. 
Kirk, Roger-E. (1996). Practical significance: A concept whose time has come. Educational \& Psychological Measurement, 56, 746-759.

Ley Orgánica 1/1990, de 3 de octubre, de Ordenación General del Sistema Educativo. BOE núm. 238, de 4 de octubre de 1990

Ley Orgánica 2/2006, de 3 de mayo, de Educación. BOE núm. 106, de 4 mayo de 2006.

Ley Orgánica 8/2013, de 9 de diciembre, para la mejora de la calidad educativa. BOE núm. 295, de 10 de diciembre de 2013.

Martín-Bris, Mario \& Gairín-Sallán, Joaquín (2007). La participación de las familias en la educación: un tema por resolver. Bordón, 59(1), 113-151.

Martínez González, Raquel-Amaya (1994). Diagnóstico de Necesidades en la Cooperación entre Familia y Centro Escolar. Proyecto realizado con la Universidad de Oviedo y el U.S.A. Department of Education, Office of Educational Research and Improvement.

Martínez-González, Raquel-Amaya, Pereira-González, Marisa, Rodríguez-Díez, Blanca, Peñadel Agua, Ana, Martínez, Rosario, García-González, Ma-Paz, Donaire-Rubio, Begoña, Álvarez, Ana-Isabel \& Casielles, Victoria (2000). Dinamización de las relaciones familia-centro escolar a través de la formación del profesorado en este campo de actuación. REOP, 11(19), 107-120.

Repáraz-Abaitua, Rosario \& Jiménez-García, Eva (2015). Padres, tutores y directores ante la participación de la familia en la escuela. Un análisis comparado. Participación Educativa, 4(7) 39-45.

Risko, Victoria \& Walker-Dalhouse, Doris (2009). Parents and teachers: Talking with or past one another. The Reading Teacher, 62(5), 442-444.

Rodríguez-Ruiz, Beatriz, Rodrigo-López, Mª-José \& Martínez-González, Raquel-Amaya (2015). Cross-Contextual Variability in Parents' and Tutors' Conflict Resolution Styles and Positive Development. The Journal of Educational Research, 108(6), 480-491.

Rodríguez-Ruiz, Beatriz, Álvarez-Blanco, Lucía, Martínez-González, Raquel-Amaya \& Epstein, Joyce (2019). Presentación del número Relación entre Centros Educativos, Familias y Entidades Comunitarias. Aula abierta, 48(1), 7-10.

Sanz-Oro, Rafael (2010). El profesor como tutor: un reto a consolidar en el ejercicio profesional de la orientación. REOP, 21(2), 346-357.

Sheldon, Steve-B. \& Epstein, Joyce-L. (2005). Involvement counts: Family and community partnerships and mathematics achievement. The Journal of Educational Research, 98(4), 196-207.

Sinclair, Marta-F., Lam, Shui-Fong, Christenson, Sandra-L. \& Evelo, David (1993). Action research in middle schools: Anwatin and Northeast Middle Schools, Minneapolis, Minnesota. Equity and Choice, 10, 22-24.

Torrego-Seijo, Juan-Carlos (Coord.) (2014). 8 ideas clave. La tutoría en los centros educativos. Barcelona: Graó. 